\title{
Tagungsbericht
}

\author{
Joachim Dinter, Dominik Hagel, Franziska Harnisch, Tabea Klaus, Miriam Kötter, Gunther \\ Kunze, Mirco Limpinsel-Pesavento, Sina Menzel, Anne Rethmann, Cosima Wagner,
} Janet Wagner

\section{Der 109. Deutsche Bibliothekartag 2021 in Bremen}

https://doi.org/10.1515/abitech-2021-0045

\section{Einleitung}

Die diesjährige Veranstaltung war aus diversen Gründen eine besondere. Nach dem pandemiebedingten Ausfall 2020 konnte in diesem Jahr ein hybrides Format angeboten werden. Dadurch konnten circa 100 Personen an zwei Tagen vor Ort in Bremen live und mehr als 2000 Interessierte online teilnehmen. Die Themen unter dem Motto „In der Fremde und Zuhause - Wagen und Gewinnen“ waren breit gestreut von neuen bibliothekarischen Handlungsfeldern über strategische Planungen in Bibliotheken bis zum Themenkomplex Bibliothek und Gesellschaft.

Die Übertragung der Veranstaltung ins vorwiegend Digitale kann als gelungen bezeichnet werden - vor allem mit Blick auf die Inhalte. Besonders praktisch ist in diesem Jahr, dass die Videoaufzeichnungen der Vorträge und Diskussion auch im Nachhinein abrufbar sind. Abstriche mussten natürlich in Bezug auf Begegnungen gemacht werden. Es ist zu hoffen, dass im nächsten Jahr eine Veranstaltung vor Ort mit allen, die das wünschen, stattfinden kann, bei der gleichzeitig die Vorteile aus diesem Jahr berücksichtigt werden.

Eine weitere Besonderheit des Kongresses 2021 war die Initiative zur Umbenennung des Veranstaltungstitels, die sich während der laufenden Veranstaltung bildete. Im Kern geht es darum, die Diversität und Vielfalt der Themen und Teilnehmenden auch in der Bezeichnung der Veranstaltung widerzuspiegeln. Aus diesem Grund wurde die Petition „Zeitgemäßer Name für den ,Bibliothekar'tag“ gestartet, die nach zwei Wochen bereits über 1500 Unterschriften erreicht hatte. ${ }^{1}$ Die Beitragenden dieses Berichts unterstützen diese Initiative. Der erste digitale Bibliothekartag könnte daher zugleich der letzte „Bibliothekartag" gewesen sein.

1 https://www.openpetition.de/petition/online/zeitgemaessername-fuer-den-bibliothekartag (17.09.2021).

\section{Neue bibliothekarische Handlungsfelder}

Die gegenwärtig stattfindenden medialen und gesellschaftlichen Veränderungen und die mit ihnen verbundenen Herausforderungen für Bibliotheken bildeten auch dieses Jahr den thematischen Rahmen großer Teile der Veranstaltung. So loteten mehrere, ganz unterschiedliche Beiträge neue bibliothekarische Handlungsfelder aus.

\subsection{OER-Strategien und Datenkompetenz}

Zunehmendes bibliothekarisches Interesse gilt beispielsweise dem Thema Datenkompetenz, dem sich die Session „OER-Strategien und Datenkompetenz“ widmete. In ihrem Vortrag zum Thema „Datenkompetenz als neue Schlüsselkompetenz - Welche Rolle haben Bibliotheken?" behandelte Simone Fühles-Ubach (TH Köln) die Frage, wie sich Bibliotheken bei der Vermittlung von Kompetenzen im Bereich Data Literacy einbringen können. Das am Institut für Informationswissenschaft der TH Köln entwickelte Mehrebenen-Modell zu den verschiedenen Aspekten der Datenkompetenz zeigte sehr eindrücklich die Komplexität des Themas und stellte dessen Relevanz als Zukunftsfeld für die Arbeit von Bibliotheken heraus.

Unter dem Titel „Ein Forschungsdatenrepositorium für den Austausch von Learning Analytics Daten“ stellte Ian Wolff (UB Magdeburg) ein Verfahren zur Analyse von Daten vor, die Lehrende und Lernende während der Nutzung von Lernplattformen erzeugen. Innerhalb des Kontexts Hochschule ließ sich der hohe Anwendungsbezug des jungen Forschungsfeldes unschwer erkennen, da die verwendeten Analysemodelle dabei helfen können, Lernprozesse und -probleme sichtbar zu machen und auf diesem Weg zum Beispiel Abbrecherquoten zu reduzieren. Ein Forschungsdatenrepositorium erweist sich in diesem Prozess als hilfreich, um Daten zu aggregieren und umfangreichere Analysen zu ermöglichen.

Im abschließenden Vortrag von Anna Arndt (SLUB Dresden) „Kooperative Entwicklung von Services zu Open 
Educational Resources an der SLUB Dresden gemeinsam mit der TU Dresden“ schwenkte der Fokus der Session auf den Bereich der offenen und nachnutzbaren Lehr- und Lernmaterialien. Auf Grundlage eines gemeinsamen Strategiepapiers etablieren beide Einrichtungen derzeit unterschiedliche Formate, um Lehrende über Open Educational Resources zu informieren und zum Mitmachen anzuregen. Die Vielzahl an Fragen aus dem Plenum unterstrich die Relevanz des Themas, machte aber auch deutlich, dass es noch an entsprechenden Anreizen für Hochschulmitglieder zum Teilen der eigenen Lehrmaterialien mangelt.

\section{2 \#digitalshift}

Neben dem intensiven Austausch $\mathrm{zu}$ allen bibliotheksbezogenen Themen mit der deutschsprachigen Fachcommunity gab es auch die Gelegenheit für einen „Blick über den Tellerrand“ nach Großbritannien. Unter dem Titel „\#digitalshift - Digitale Transformation und Openness in deutschen und britischen wissenschaftlichen Bibliotheken" hatten der Verein Deutscher Bibliothekarinnen und Bibliothekare (VDB) und der Fachverband Research Libraries UK (RLUK) eine Diskussionsveranstaltung organisiert, die zugleich als Auftakt für einen weiteren Austausch zwischen beiden Ländern dienen sollte. Moderiert von Ewald Brahms (UB Hildesheim) berichteten Torsten Reimer (Leiter der Abteilung „Content and Research Services“ der British Library) und Matthew Greenhall (stellvertretender Präsident von RLUK) über die „Digital-Shift"-Initiative der britischen Forschungsbibliotheken. Bereits im vergangenen Jahr hatte RLUK mittels eines Strategieprozesses ein Digital Shift Manifesto ${ }^{2}$ mit dem Ziel entwickelt, sich als wissenschaftliche Bibliotheken aktiver in die Gestaltung des Aufgabenfelds der „digitalen Transformation“ einzubringen statt sich davon treiben zu lassen. Im Manifest werden dazu für Bibliotheken insbesondere die stärkere Entwicklung von Fähigkeiten zur Technikfolgenabschätzung, mehr Kenntnisse im Bereich Künstliche Intelligenz und die Stärkung der digitalen Gestaltung von Geschäftsprozessen hervorgehoben. Dabei sollte jedoch das Vertrauen der Nutzenden in Bibliotheksinfrastrukturen nicht dadurch verspielt werden, dass mehr und mehr „Closed Systems“ von kommerziellen Anbietern in Bibliotheken verwendet würden, die zum Beispiel ein Datentracking ermöglichen, und das Gegenteil von Transparenz und ansonsten viel beschworener Offenheit darstellten.

2 https://www.rluk.ac.uk/digital-shift/ (17.09.2021).
Selbstkritisch merkte Greenhall in seiner Präsentation zu den Auswirkungen der COVID-19-Pandemie auf die RLUK-Bibliotheken an, dass sie trotz des „Digital-Shift“-Strategieprozesses weniger auf die digitalen Anforderungen vorbereitet gewesen seien, als bei der Erstellung des Manifests angenommen. „Digital Poverty“ und der ungleiche Zugang zu digitalen Informationen seien nun stark in den Fokus gerückt, aber auch Chancen wie die Entwicklung von neuen Serviceformaten, von denen er eines am Beispiel der Virtual Reading Rooms (VRR) näher erläuterte. Diese seien zunächst als pragmatische Lösung für die Unterstützung von Forschenden eingerichtet worden, damit diese mittels Video-Konferenztechnologie und Scannern, die individuell von Bibliotheksmitarbeitenden für die jeweiligen Forschenden vor Ort bedient wurden, auf nicht-digitalisierte Medien aus Bibliotheken zugreifen zu können. Mit derartigen Formaten beweise die Bibliothek ihre rasche Reaktionsfähigkeit und Serviceorientierung für ihre Nutzenden, in Großbritannien kämen diesen „Embedded Services“ daher auch zukünftig eine wichtige Bedeutung zu.

In der anschließenden Diskussion wurde das Thema der Marktmacht von kommerziellen Unternehmen in wissenschaftlichen Bibliotheken noch einmal aufgegriffen. Wichtig sei es, Grundprinzipien zum Beispiel für die Lizenzierung von Medien festzulegen, die bei jedem Beschaffungsprozess bezüglich Datensicherheit usw. angewendet werden sollten. Bibliotheken sollten sich ihrer Marktmacht stärker bewusstwerden und international auf diesem Gebiet zusammenarbeiten.

\subsection{Nachhaltigkeit}

Das Thema ökologischer, ökonomischer und sozialer Nachhaltigkeit, das im Digital Shift Manifesto ebenfalls adressiert wird, war auch Gegenstand einer weiteren Session. Neben der vom Deutschen Bibliotheksverband (dbv) dargestellten Übersicht zur Positionierung der Bibliotheken zur Agenda 2030 und den 17 Zielen für nachhaltige Entwicklung wurden mehrere Praxisbeispiele vorgestellt.

Patricia Fasheh (Stadtbücherei Schwarzenbek) und Susanne Brandt (Büchereizentrale Schleswig-Holstein) gaben Einblicke zur Vernetzung lokaler Bibliotheken. Seit letztem Jahr gibt es den „Runden Tisch Grüne Bibliotheken in Schleswig-Holstein“. ${ }^{3}$ Neben der Bündelung gemeinsamer Aktivitäten wie die Teilnahme an den jährli-

3 https://www.bz-sh.de/leistungen/fortbildungen-und-veranstaltun gen/runder-tisch-gruene-bibliotheken (17.09.2021). 
chen „Aktionstagen Nachhaltigkeit“ im September wurde dazu aufgerufen, die „bibliothekarische Blase“ zu verlassen und proaktiv lokale Zusammenarbeit anzubahnen. Eindrückliche und nachhaltige Projekte, die aus diesem Ansatz bereits entstanden, sind: die mobile Saatgutbibliothek zum Erhalt alter Saatgutsorten, das Projekt „Erzählwege“ und auch die vielfältigen Angebote zur „Bibliothek der Dinge“ in den einzelnen Bibliotheken, die das grundlegende Prinzip „Leihen statt Kaufen“ als eine Strategie der nachhaltigen Entwicklung unterstützen und fördern.

Der Ermittlung der eigenen Klimabilanz widmeten sich die Stadtbibliothek Berlin-Pankow und die Stadtbücherei Norderstedt. Gefördert als Projekt der Kulturstiftung des Bundes wurden von Herbst 2020 bis Januar 2021 alle direkten und indirekten Treibhausgasemissionswerte in den Bereichen Strom- und Energieverbrauch, Mobilität, Fuhrpark, Abfall und Müll und Elektroschrott innerhalb der Einrichtung ermittelt und ausgewertet. Tim Schumann (Stadtbibliothek Berlin Pankow) berichtete von einem entwickelten Maßnahmenkatalog zur konkreten Einsparung von Emissionen. Klimabilanzierungen seien ein erster und wichtiger Schritt für Bibliotheken, um interne und externe Prozesse in der eigenen Einrichtung hinsichtlich Ressourceneinsparungen und Emissionsreduzierung anzustoßen. Die validen Werte einer Klimabilanz können dazu beitragen, im eigenen Bibliotheksteam für das bedeutende Thema Nachhaltigkeit zu sensibilisieren und ein Umdenken herbeizuführen.

\subsection{Sprachenvielfalt}

In dem von Miriam Schmidt (Stadtbibliothek Magdeburg) und Britta Schmedemann (Stadtbibliothek Bremen) organisierten und moderierten Themenkreis „Politische und gesellschaftliche Sprachenvielfalt und Digitalisierung“ lag der Fokus auf der Rolle digitaler Medien für den (Zweit-)Sprachenerwerb von Kindern, sowie am Rande auch um deren Einsatz in der Erwachsenenbildung. Den theoretischen Überbau lieferte Marija Smuda Duric (Internationale Forschungsstelle für Mehrsprachigkeit der Ludwig-Maximilians-Universität München), die gegen das Zerrbild des defektiven Spracherwerbs argumentierte, der gerade Kindern unterstellt wird, deren Anderssprachigkeit als besonders „markiert“ gelten kann: ${ }^{4}$ Die Vielfalt

4 Also solche Kinder, deren Erstsprache nicht Englisch, Französisch oder möglicherweise eine andere (westeuropäische) Sprache ist, deren Beherrschung/Verwendung mit einem gewissen Prestige ausgestattet ist. an sprachlichem Input zahle sich in jedem Fall aus und habe keine negativen Auswirkungen auf das Erlernen der Mehrheitssprache. Es gehe vor allem um eine altersgerechte mehrsprachige Medienversorgung. Dabei können Bibliotheken wichtige Hilfestellung leisten. Wie diese aussehen kann, wurde in der Folge vorgestellt. Krystyna Strozyk und Gudrun Möwes-Butschko berichteten von der Ein- und Weiterführung des Projekts Mulingula, ${ }^{5}$ das multilinguale Leseaktivitäten im analogen und digitalen Raum verknüpft. Darüber hinaus präsentierte Silke Schumann (Stadtbücherei Frankfurt am Main) unterschiedliche bereits existierende Plattformen und Werkzeuge, ${ }^{6}$ die Bibliotheken zur multilingualen Leseförderung einsetzen können. Bei der Auswahl wurde besonderer Wert auf die sprachliche Authentizität und Nähe zur Lebensrealität der Kinder gelegt. Die Bedeutung der Leseförderung liegt auf der Hand: Solche Maßnahmen können entscheidend zur Inklusion bildungsbenachteiligter und/oder sozial stigmatisierter Gruppen beitragen und stellen daher eine essentielle Dienstleistung öffentlicher Bibliotheken dar. Dennoch ist die Intersektion von Digitalisierung und Multilingualität auch für wissenschaftliche Bibliotheken ein relevantes Feld (Beispiel: Forschungsdatenmanagement) und bedarf in Zukunft sicherlich größerer Aufmerksamkeit.

\subsection{Co-Working}

Klaus Ulrich Werner (UB Freie Universität Berlin) sprach über Co-Working-Spaces und die davon ausgehenden Inspirationen für den Lernort Bibliothek. Das Selbstverständnis von Bibliotheken als Lernort hat sich grundlegend gewandelt. Zunehmend sind Bibliotheken lebendige, öffentliche Orte, die mit neuen Raum-Ideen den sehr unterschiedlichen Bedürfnissen der Nutzerinnen und Nutzer gerecht werden wollen. Zusätzlich zum traditionellen „stillen Arbeitsplatz“ einerseits und kommunikativen Zonen andererseits gehören heute geschützte Räume für kleine Arbeitsgruppen, Schulungsräume sowie multifunktionale Flächen für kreative Aktivitäten wie zum Beispiel Makerspaces zum Profil von Bibliotheken. Co-Working-Räume können jedoch noch mehr bieten für das gemeinsame Arbeiten: eine durchgängige (online) Betreuung für die IT-Infrastrukturen, eine Bar mit Selbstbedienung zu Kaffee, Tee und Softdrinks oder auch Ein-

5 https://www.mulingula-praxis.de/ (17.09.2021). 6 Netzwerk GDL (Global Digital Library): https://digitallibrary.io; Bilingual Picturebooks: https://www.bilingual-picturebooks.org/; Amira: https://www.amira-lesen.de/\#page=home (alle 17.09.2021). 
richtungsgegenstände wie ein Teppich. Zusätzliche Räume für die Entspannung mit Hängesesseln, Tischkicker oder Bewegungszonen können das Co-Working-Konzept in seiner Gänze abrunden. Der klassische Lesesaal ist weiterhin gewünscht, reiht sich jedoch zukünftig in die verschiedenen anderen Raumangebote von Bibliotheken ein.

\subsection{Zum Status quo des Fachreferats}

Wie sich, auch angesichts all dieser Entwicklungen, das traditionelle Fachreferat entwickelt, war Gegenstand einer von Vivien Petras (Humboldt-Universität zu Berlin) moderierten Diskussionsveranstaltung. Bei der von den beiden VDB-Kommissionen für forschungsnahe Dienste und für Fachreferatsarbeit organisierten Veranstaltung kamen sowohl Fachreferentinnen und Fachreferenten (Björn Gebert, Fachreferent für Geschichte, Theologie und Kunst an der ULB Münster, und Sibylle Hermann, Fachreferentin für Maschinenbau an der UB Stuttgart) als auch eine Bibliotheksleiterin (Maria Elisabeth Müller, Direktorin der SuUB Bremen) und ein Wissenschaftler (Albrecht Hausmann, Professor für kulturwissenschaftliche Mediävistik an der Carl von Ossietzky Universität Oldenburg) zu Wort. Deutlich wurden dabei zunächst die Breite und fachspezifisch sehr unterschiedliche Akzentuierung der forschungsnahen bibliothekarischen Arbeit. Der Anteil von Tätigkeiten, die dem Fachreferat klassischer Prägung zugerechnet werden können, ist teilweise so gering, dass selbst der Terminus „Fachreferat“ in Frage gestellt werden muss. Bei allen Unterschieden in den verschiedenen Disziplinen zeigte die Diskussion, dass die wesentliche Komponente der Tätigkeit von einzelnen Fächern dezidiert zugeordneten Bibliothekarinnen und Bibliothekaren eine Übersetzungs- und Vermittlungsfunktion zwischen dem Dienstleistungsportfolio der Bibliotheken und dem wissenschaftlichem Personal ist. Die Diskussion verlief insofern unglücklich, als sich die Diskutierenden hierfür auf die Metapher des Brückenkopfes (d.i. im Wortsinn eine militärische Stellung auf feindlichem Territorium) einigten. Besonders erhellend für die bibliothekarische Gemeinschaft sollten die Beiträge Albrecht Hausmanns sein, der aus der Perspektive des Wissenschaftlers auf die Schwierigkeiten der Interaktion zwischen Forschenden und Bibliotheksmitarbeitenden hinwies und klarmachte, dass offene Kommunikation und eine ,verwandte wissenschaftliche Sozialisation“ Voraussetzung für eine produktive Zusammenarbeit ist. Die Podiumsdiskussion bot Ansätze zur Vermessung eines komplexen Zusammenhangs, der im Rahmen eines o-bib-Themenheftes weiter ergründet werden soll.

\subsection{Open Access und Open Science}

Open-Access-Dienstleistungen sind inzwischen Teil des Kerngeschäfts von wissenschaftlichen Bibliotheken und damit jedes Jahr ein Thema von großem Interesse. 2021 waren drei Sessions im Themenkreis „Content und Digitalisierung" den Fragen rund um Open Access in Bibliotheken gewidmet.

Den Beginn machte am Mittwochmorgen die Session „Open Access - Chancen und Risiken“. Angela Holzer (Deutsche Forschungsgemeinschaft - DFG) stellte darin die beiden neuen DFG-Förderprogramme aus dem OpenAccess-Bereich vor: „Infrastrukturen für wissenschaftliches Publizieren“ und „Open-Access-Publikationskosten“. Letzteres gewährt einen Zuschuss zu Publikationskosten wissenschaftlicher Einrichtungen und soll damit die Transformation hin zu einer publikationsbasierten Finanzierung von Open-Access-Artikeln unterstützen. 2021 war die Anzahl der Anträge höher als von der DFG erwartet und das Antragsvolumen ist entsprechend überzeichnet. Die entstehenden Mehrkosten für Open-Access-Publikationen können nicht von der DFG allein getragen werden. Abschließend stellte Angela Holzer die Rahmenbedingungen für eine nachhaltige Open-Access-Transformation vor und ging dabei auch genauer auf die wesentliche Rolle der Bibliotheken als Vermittlung zwischen Forschenden und Verlagen ein.

Im Anschluss folgte der Vortrag „Open-Access-Transformationsverträge - Entwicklungen in Deutschland und international“ von Kai Geschuhn (Max Planck Digital Library - MPDL). Zunächst wurden die Hintergründe von Transformationsverträgen vorgestellt und anschließend auf die von der MPDL gegründete ESAC Initiative (Efficiency and Standards for Article Charges) eingegangen, ${ }^{7}$ die einen internationalen Austausch zu diesem Thema ermöglicht.

Den Abschluss der Session bildete der Vortrag „Das Lesen der Anderen: User Tracking auf Verlags-Plattformen" von Renke Siems (Baden-Württembergisches Ministerium für Wissenschaft und Kunst). Siems berichtete über den rasanten Anstieg von Data Analytics durch Großverlage. Das User-Tracking stellt ein finanziell bedeutendes neues Geschäftsfeld für die Verlage dar. Ob und wie Datenprodukte weiterverkauft oder -verwendet werden, ist mangels Transparenz jedoch weitgehend unklar. Passend wurde die aktuelle Situation daher als ein neues Modell von Double Dipping beschrieben: „Autor`innen bezahlen mit APCs, Nutzer`innen mit ihren persönlichen Daten“. Um dieser Entwicklung entgegenzuwirken, werden drin-

7 https://esac-initiative.org/ (17.09.2021). 
gend regulatorische Maßnahmen benötigt. Diese Forderung unterstützt auch der Ausschuss für Wissenschaftliche Bibliotheken und Informationssysteme (AWBI) der DFG in einem Informationspapier zum Datentracking in der Wissenschaft. ${ }^{8}$

Am Donnerstag folgte eine Podiumsdiskussion zum Thema „Wie finanzieren wir die Open-Access-Transformation?" geleitet von Klaus-Rainer Brintzinger (UB der Ludwig-Maximilians-Universität München). Ursprünglich schon als zentrale Veranstaltung für den Bibliothekartag 2020 geplant, wurde diese Diskussion nun aufgrund der aktuell steigenden Bedeutung ins Digitale verlegt. Anwesend vor Ort auf dem Podium waren der Generalsekretär der Hochschulrektorenkonferenz Jens-Peter Gaul, Konstanze Söllner (UB Erlangen-Nürnberg) und Ralf Brugbauer (UB Bayreuth). Jens-Peter Gaul stellte zunächst die Erfolge der DEAL-Verhandlungen vor, worauf ein Bericht über die Erfahrungen der beiden Bibliotheken folgte. Dabei standen die Nachzahlungen im Rahmen der DEAL-Verträge im Mittelpunkt, die aufgrund der Differenz der vereinbarten Zahlungen und der Anzahl der veröffentlichten Artikel beim jeweiligen Verlag resultieren. Der von der Universität Erlangen-Nürnberg geforderte Nachzahlungsbetrag war so hoch, dass er nicht geleistet werden kann und auch im Bereich der Gold-Open-Access-Journals scheint es aufgrund von steigenden Artikelzahlen keine Kostenkontrolle mehr zu geben. Es sei daher im Moment unklar, ob die Universität die Kosten für DEAL zukünftig tragen kann. Auch Ralf Brugbauer betrachtet die Entwicklungen mit Sorge. Nachdem wie zuvor berichtet das DFG-Förderprogramm „Open-Access-Publikationskosten“ überzeichnet ist, sei es ungewiss, wie die Kosten für die DEAL-Verträge in Zukunft aufgebracht werden können. Darüber hinaus wurde mehr Transparenz in Bezug auf die zu leistenden Zahlungen gefordert.

In der Sektion „Auf dem Weg zu mehr Openness“ ging es um verschiedene Perspektiven der bibliothekarischen Arbeit in Zeiten von Open Science, wobei der rote Faden zwischen den einzelnen Slots nicht immer erkennbar war. Diskussionsanregend war der Beitrag von Marcel Knöchelmann (University College London), der über „Das gerechtere Publizieren und die Bibliothek der Zukunft: Über nationale Transformationen und deren lokale Verwirklichungen“ referierte. Marcel Knöchelmann vermisst eine Debatte darüber, wie wissenschaftliches Publizieren in Zukunft aussehen soll und warf Bibliotheken vor, sich durch die Versteifung auf Open Access an der Engführung der Diskussion zu beteiligen. Geboten sei es aber vielmehr,

8 https://www.dfg.de/download/pdf/foerderung/programme/lis/ datentracking_papier_de.pdf (17.09.2021).
Offenheit nicht als Ziel, sondern als Basis zu verstehen, von der aus die Rolle der Bibliothek im Wissenschaftskontext neu verhandelt werden könne.

Juliane Stiller (Agentur You, We \& Digital) stellte in ihrem Vortrag erste Ergebnisse einer Online-Konsultation vor, die in Zusammenarbeit mit dem Open-Access-Büro Berlin an Wissenschafts- und Kultureinrichtungen des Landes durchgeführt wurde. Identifiziert werden sollten an den Einrichtungen bereits bestehende Praktiken und Einstellungen zum Themenkomplex Open Research, um ein Bewusstsein für größtmögliche Offenheit in allen Phasen des Forschungszyklus zu schaffen und eine Grundlage für die Formulierung einer Berliner Open-Science-Strategie zu legen.

Aus der Arbeit an der Digitalisierungsstrategie des Bundes für den Kulturbereich berichtete Frédéric Döhl (DNB) in seinem Projektbericht. Der Anspruch des sparten- und institutionsübergreifenden Strategieprojekts ist es, kulturelle Arbeit im digitalen Wandel sichtbar zu machen und prioritäre Arbeitsfelder des öffentlichen Kulturbereichs für die kommenden Jahre zu umreißen.

Zum Abschluss der Session wurde im Beitrag von Elisabeth Engl (Herzog August Bibliothek Wolfenbüttel) der Fokus auf die technische Umsetzung gelegt. Das von der DFG geförderte Vorhaben OCR-D zur Digitalisierung von Drucken aus dem 16.-19. Jahrhundert geht in seine mittlerweile dritte Projektphase und macht den Schritt von der Pilotphase in die bibliothekarische Praxis. Dargestellt wurden Herausforderungen beim Rollout der Software und die vielfältigen Anwendungsszenarien bei der Massendigitalisierung. Eine User-Community soll zukünftig Unterstützung im täglichen Betrieb leisten.

\section{Strategische Planung in Bibliotheken}

Großes Interesse erhielten die Fragen der strategischen Planung und des Managements, konkret also die Frage: Wie können Bibliotheken als Organisationen auf Herausforderungen jeglicher Art reagieren.

\subsection{Projektplanung und strategisches Management}

In der Session „Projektplanung und strategisches Management“ präsentierte Olaf Eigenbrodt (SUB Hamburg) fünf Thesen zum Verhältnis aktueller bibliothekarischer Herausforderungen und bibliothekswissenschaftlicher 
Antwortangebote und gab damit den folgenden Vorstellungen aus der Bibliothekspraxis einen intellektuell anregenden Rahmen. Olaf Eigenbrodts Aufriss behauptete (1) die Notwendigkeit sowohl historischer Reflexion als auch (2) soziologischer Information bibliothekarischen Handelns, adressierte (3) die Verhandlung ethischer Grundlagen ebenso wie (4) die Theorie von Organisations- und Managementkulturen und wies (5) auf die nicht-technischen Aspekte der Digitalisierung hin - womit ein sehr vielseitiges Themenangebot dargelegt wurde, das in den einzelnen Vorträgen nur teilweise aufgegriffen werden konnte.

Dass der von Olaf Eigenbrodt skizzierte Problemkomplex in der Praxis sehr verschieden skaliert werden muss, machte der Vortrag von Michaela Jobb (Bibliothek Wirtschaft und Management der TU Berlin) deutlich, der das Managementinstrument des Retreats zur Einbindung der Mitarbeitenden in die Projektplanung und gleichzeitigen Entlastung der Bibliotheksleitung vorstellte.

Über eine ungewöhnliche Zusammenarbeit berichteten Christian Osterheld (ZB Zürich) und Benjamin Scher (Universität St. Gallen), die gemeinsam versuchen, durch die digitale Transformation bewirkte Verunsicherungen der Bibliothek produktiv zu machen. Zu diesem Zweck wurde 2018 eine Forschungspartnerschaft zwischen der ZB Zürich und dem RISE Management Innovation Lab der Universität St. Gallen ins Leben gerufen, in deren Rahmen Benjamin Scher als teilnehmender Beobachter den Strategieentwicklungsprozess der Bibliothek begleitet und unterstützt.

Martin Lee und Christina Riesenweber (UB der Freien Universität Berlin) berichteten vom Strategieentwicklungsprozess der Universitätsbibliothek der Freien Universität Berlin, der das Bibliothekssystem mit dem bis dahin unabhängigen Center für digitale Systeme der Universität zusammenführte. Der Schwerpunkt wurde dabei auf eine nutzerorientierte und wandlungsfähige Ausrichtung der Gesamtorganisation gelegt. Basierend auf einem systemischen, der Komplexität der Umwelten Rechnung tragenden Ansatz wurde in einem extern begleiteten, partizipativen Prozess das Konzept einer zukunftsfähigen Universitätsbibliothek entwickelt, in der nicht zuletzt die Organisationsentwicklung einen festen Platz innerhalb der Organisation hat.

\subsection{Fehlerkultur}

In der von Frank Scholze (DNB) moderierten Veranstaltung unter dem Titel „Dumm gelaufen“ wurden „Beispiele des produktiven Scheiterns zum Aufbau von Fehlerkom- petenz und Feedbackkultur" vorgestellt und diskutiert. Während Scheitern nach wie vor häufig als Hinderungsgrund gesehen wird, Projekte anzugehen, verstanden die Referentinnen und Referenten - Martin Lee (UB der Freien Universität Berlin), Caroline Leiß (UB der Technischen Universität München) Wolfgang Stille (hessian.AI - Hessisches Zentrum für Künstliche Intelligenz), Frauke Untiedt (Stiftung Hamburger Öffentliche Bücherhallen) und Danilo Vetter (Stadtbibliothek Berlin Pankow) - Fehler als notwendiges und potentiell chancenreiches Moment jeglicher Projektarbeit, insbesondere wenn es darum geht, neue Formate zu entwickeln. Die verbreitete Angst, Fehler zu machen, habe einen großen Anteil daran, dass insbesondere der Öffentliche Dienst weitgehend agilitätsfeindlich sei. Die Referierenden forderten dementsprechend neue Kulturen des Sprechens über Fehler und stellten exemplarisch Projekte vor, die gescheitert sind. Aus diesem Scheitern aber, darin stimmten alle überein, haben sie Entscheidendes gelernt.

\subsection{Einbeziehung der Nutzenden}

Ein Thema, das sich bereits 2019 in vielen Vorträgen zeigte, war in diesem Jahr noch deutlicher zu erkennen: die Einbeziehung Nutzender für eine menschzentrierte Weiterentwicklung von Bibliotheken. Als zentrale Begriffe fielen hier immer wieder „Benutzungsforschung“ und „Partizipation“.

Dass Kompetenzen auf diesem Gebiet einen eindeutigen Mehrwert und neue Möglichkeiten in Bibliotheken bringen, beschrieb Romy Hilbrich in ihren Beiträgen auf dem Podium „Kundenorientierung quergedacht: Neue Kompetenzen für neue Services (?)“ des dbv eindrücklich. Sie bekleidet seit 2018 die Stabsstelle für „Nutzerforschung und Statistik" an der Staatsbibliothek zu Berlin, die dort bei der Leitung der Benutzungsabteilung angesiedelt ist.

Auch Claudius Lüthi (ZB Zürich) griff im Block „Agil und smart im Team" die Rolle Benutzungsforschung für die Produktentwicklung auf. Der Ansatz „Lösung sucht ein Problem“ gehe hier „immer schief“. Man müsse vielmehr durch Forschung die Bedürfnisse der Nutzenden erkennen und dafür Lösungen suchen. Empirie sei hier höher zu bewerten als bloße Annahmen.

Im selben Block berichteten Linda Freyberg (Urban Complexity Lab der Fachhochschule Potsdam) und Sabine Wolf (Stadtbibliothek Berlin-Mitte) von Partizipation an Bibliotheken als Smart Libraries. Im Kontrast zum Begriff der „Kooperation“ hoben sie hervor, dass „Partizipation“ die Gleichwertigkeit der Partnerinnen und Partner voraussetze, jedoch verschiedene Ausprägungen haben könne. 
Die Partizipationspyramide ${ }^{9}$ biete hier einen Überblick über verschiedene Grade der Beteiligung, die von Informieren hin zu Eigenaktivitäten der Nutzenden reiche.

Martin Holtorf und Jörg Räuber beschrieben die neuesten Aktivitäten der DNB im Bereich Benutzungsforschung. Eine aktuelle qualitative Studie aus dem September 2020 bezog sich dabei auf die Frage, wie sich die Aussetzung der Nutzungsgebühren und die Corona-Lage individuell auf Vor-Ort Nutzende der DNB auswirkt. In Kurzinterviews wurden dabei viele positive, aber auch einige negative Auswirkungen der fehlenden Nutzungsgebühren strukturiert erfasst. Eine erste praktische Auswertung der Interview-Ergebnisse ist die Anpassung der buchbaren Zeitslots für Arbeitsplätze mit Hygienekonzept nach Wiedereröffnung. Parallel fand 2020 die aktuellste quantitative Nutzendenbefragung der DNB statt. Auch hier stellten sich im Vergleich zur letzten großen Befragung 2016 einige neue Erkenntnisse heraus, die oftmals auch mit den veränderten Nutzungsbedingungen während der Pandemie zusammenhingen.

Auch im Bereich Öffentlicher Bibliotheken wurde die Einbeziehung Nutzender immer wieder in Projektvorstellungen deutlich. Die Vorträge der beiden Blöcke „Lernräume im Wandel I + II"10 zeigten zum Beispiel, dass partizipative Prozesse bei der Um- oder Neuplanung von Bibliotheksbauten eine immer wichtigere Rolle spielen. Das betonte Ragna Körby (Stadtplanerin von der TU Kaiserslautern) und zeigte viele anschauliche Beispiele für aktuelle Entwicklungen von Bibliotheken im Stadtbild.

\section{Bibliothek und Gesellschaft}

Die Zeit der COVID-19-Pandemie ist auch durch den spürbaren Zuwachs von Verschwörungstheorien, Fake News und Rechtspopulismus gekennzeichnet. Gleich mehrere Beiträge setzen sich mit diesem Problemkomplex auseinander.

\subsection{Framework for Information Literacy for Higher Education}

Anlässlich der kürzlich erschienenen deutschen Übersetzung des „Framework for Information Literacy for Higher Education” gab es eine Podiumsdiskussion mit

9 www.partizipationspyramide.de (17.09.2021).

10 Siehe auch die Zusammenfassungen einzelner hier beschriebener Vorträge als Blogbeitrag unter https://b-u-b.de/bibliothekartag2021-blog/ (17.09.2021). dem Bibliothekar und Mit-Übersetzer Oliver Schoenbeck (Bibliotheks- und Informationssystem der Universität Oldenburg), dem Bildungswissenschaftler Karsten Speck (Universität Oldenburg) und dem Informationswissenschaftler Joachim Griesbau (Universität Hildesheim). Auch wenn es Details zu kritisieren gäbe und die Übertragung vom angloamerikanischen auf den deutschen Kontext nicht immer reibungsfrei möglich sei, begrüßten die Diskutanten das Framework doch prinzipiell. Gerade aktuell sei die Förderung des Hinterfragens und Abwägens von Informationen im Rahmen der Informationskompetenz eine wichtige Aufgabe, auch und gerade in Bibliotheken.

\subsection{Citizen Science}

Vielfältige Möglichkeiten für Bibliotheken, ein Gegengewicht $\mathrm{zu}$ Verschwörungstheorien und sogenannten alternativen Fakten zu setzen, liegen in den verschiedenen Formaten von Citizen Science, der Beteiligung von Laien an wissenschaftlichen Forschungsprojekten. Thomas Kaarsted (University of Southern Denmark) hielt ein enthusiastisches und inspirierendes Plädoyer für das Engagement von Bibliotheken im Zusammenhang mit Citizen Science. Bibliotheken könnten als Hubs für Citizen Science das Bindeglied zwischen den Bürgerinnen und Bürgern, Wissenschaftsinstitutionen, Schulen und der Politik sein. Insbesondere als Beispiele für die Umsetzung der Sustainable Development Goals der Vereinten Nationen existierten in Bibliotheken in Deutschland bereits zahlreiche Citizen Science nahestehende Projekte. ${ }^{11}$ Thomas Kaarsted ermutigte die Bibliotheken, dieses Engagement zukünftig fortzusetzen und auszubauen.

Die beiden Wissenschaftlerinnen Melike Peterson (Universität Bremen) und Katja Thiele (Universität Bonn) nahmen Citizen Science aus der Perspektive der Forschung für und mit Bibliotheken in den Blick. Citizen Science als partizipativer Forschungsstil bereichere durch neue Impulse die Wissenschaftskommunikation und fordere etablierte Methoden, Prozesse und Hierarchien heraus.

Stefan Wiederkehr (ZB Zürich) berichtete von den Chancen von Citizen Science für Bibliotheken. Vor allem für Bibliotheken mit einem besonderen historischen, lokalen Bestand böten sich im Zusammenhang mit der Digitalisierung nicht nur vielfältige Möglichkeiten der unterstützenden und forschenden Beteiligung der Citizens den Kundinnen und Kunden der Bibliothek. Durch Citizen Science entstünden einerseits neue Fragestellungen an den Bestand und zugleich gewährleisteten die Infrastruk-

11 https://www.biblio2030.de/beispielsammlung/ (17.09.2021). 
turen der Bibliotheken die nachhaltige Bereitstellung der Ergebnisse aus den Projekten - eine vielversprechende Verbindung.

\subsection{Riffreporter}

Die seit 2014 laufende Masterclass Wissenschaftsjournalismus ermöglicht es, neue Formate in und mit Bibliotheken zu entwickeln. In der Veranstaltung „Journalismus in die Bibliothek. Die Riffreporter-Masterclass 2020“ berichteten zwölf Journalistinnen und Journalisten exemplarisch von ihren Projekten. Sie nutzen bibliothekarische Räume, um den Nutzenden ganz unterschiedliche Themen zu vermitteln. Indem sie teils ihren Schreibtisch für einige Wochen in der Bibliothek platzieren, soll zudem die journalistische Arbeit transparenter werden. Auch für diese Form der Zusammenarbeit bieten Bibliotheken eine ideale Umgebung.

\subsection{Medien an den Rändern}

Um ein dezidiert bibliothekarisches Problem im Zusammenhang mit Verschwörungstheorien und Fake News ging es in der Vorstellung von „Medien an den Rändern“: Wie sollen Bibliotheken mit Medien umgehen, die „an den Rändern“ lokalisiert sind, die also beispielsweise rechtspopulistische Inhalte haben? Einigen technischen Störungen zum Trotz skizzierten vor allem Tom Becker (TH Köln) und Beate Meinck (Stadtbibliothek Reutlingen), mit welchen konzeptuellen Fragen und Probleme sich die noch junge Arbeitsgruppe befasst. Im Chat entspann sich eine lebhafte Diskussion über anzuwendende Kriterien. Der „Expert:innenzirkel“ ist, das machte Tom Becker deutlich, offen für die Beteiligung weiterer Kolleginnen und Kollegen.

\subsection{Provenienzforschung}

Im Gegensatz zur Existenz von NS-Raubgut im Kunsthandel und in Museen ist der breiten Öffentlichkeit weit weniger bekannt, dass der Raub von Kulturgütern im großen Umfang auch Bücher betraf. Es ist daher sehr begrüßenswert, dass dieser Thematik mit drei Veranstaltungen gebührend Platz eingeräumt wurde. In der ersten Veranstaltung „Provenienzen in der Praxis“ standen Berichte aus dem Arbeitsalltag der Provenienzforschung im Vordergrund. Hannah Schneider berichtete von ihrer Arbeit im Archiv der sozialen Demokratie der Friedrich-
Ebert-Stiftung. Das vom Deutschen Zentrum Kulturgutverluste finanzierte Projekt beinhaltet die Überprüfung von etwa 18000 projektrelevanten Büchern. Ziel der systematischen Bestandsüberprüfung ist die virtuelle Rekonstruktion der historischen SPD-Parteibibliothek vor 1933.

Sebastian Finsterwalder (ZLB Berlin) unterstrich in seinem Vortrag, dass der offene Umgang mit erhobenen Forschungsdaten unerlässlich ist für eine erfolgreiche Provenienzforschung. Forschungsdaten wie Autogramme, Widmungen, Exlibris werden direkt nach der Erfassung online in die Provenienzdatenbank „Looted Cultural Assets" gestellt. Anhand der Identifikationsgeschichte des Exlibris von Ernst G. Preuß zeigte Sebastian Finsterwalder, wie wichtig sowohl die Veröffentlichung von Provenienzdaten als auch die Inanspruchnahme von Hinweisen aus der Bevölkerung für die eigene Arbeit ist.

Tomasz Łopatka berichtete von seiner Katalogisierungsarbeit in der Forschungsbibliothek des Herder-Instituts. Zwischen 2016 und 2019 wurde ein Teilbestand, der aus der ehemaligen Publikationsstelle Berlin-Dahlem stammte, auf Provenienzspuren hin untersucht. Es handelte sich um 17500 Bände, die unter NS-Raubgutverdacht standen. Die erste Restitution sei in Planung. Das Herder-Institut erstellte eine eigene Access-Datenbank. Mittlerweile sind circa 11000 Titel im eigenen Katalog mit Provenienzmerkmalen versehen und mit der GND verknüpft. Die Moderatorin Regine Dehnel fasste die Beiträge des Panels treffend zusammen: Bei der Provenienzforschung gehe es um Öffentlichkeit, Transparenz, Vernetzung und nicht zuletzt um die Erinnerung an die Menschen, denen die Bücher geraubt wurden.

Diese Aspekte wurden in der Podiumsdiskussion „Provenienzforschung - Und was haben wir davon?“ weiter diskutiert. Ulrich Johannes Schneider (UB Leipzig) moderierte die Runde mit Christiane Hoffrath (USB Köln), Robert Langer (Sächsische Landesfachstelle für Bibliotheken) und Regine Dehnel (Staatsbibliothek zu Berlin). Ulrich Johannes Schneider hob dabei hervor, dass Bibliotheken mit der eigenen Provenienzforschung einen großen Beitrag für weitere Forschungen leisten. Regine Dehnel griff diesen Gedanken auf und verwies darauf, dass Provenienzforschung andere Forschungen erst ermöglicht wie Institutionengeschichte, Buchgeschichte und Biographien. Bei Provenienzforschung gehe es, so Christiane Hoffrath, auch nicht allein um Wiedergutmachung, sondern um Forschung, Dokumentation und Restitution. Das sei kein Nice-to-Have, keine moralische Frage, sondern eine zentrale Aufgabe von Bibliotheken, Archiven und Museen, die seit 1999 mit der Gemeinsamen Erklärung zudem auch eine Verpflichtung ist. Robert Langer, der zuvor in der Provenienzforschung in der Stadtbiblio- 
thek Bautzen arbeitete, wies auf die Schwierigkeit hin, ein Bewusstsein für NS-Raubgut in Bibliotheken zu schaffen, wenn keine Infrastruktur vorhanden ist. In seiner derzeitigen Position als Leiter der Sächsischen Landesfachstelle für Bibliotheken sieht er in dem Vernetzungsaspekt eine wesentliche Aufgabe. Die Veranstaltungen haben verdeutlicht, dass Provenienzforschung 1. ein selbstverständlicher Bestandteil bibliothekarischen Arbeitens sein sollte, 2. der Kooperation und des freien Zugangs zu Forschungsdaten bedarf und 3. Erinnerung an diejenigen Menschen ermöglicht, denen nicht nur die Bücher, sondern in den meisten Fällen auch ihre Leben brutal genommen wurden. In Zeiten, in denen der Ruf nach einem Schlussstrich in Deutschland wieder lauter wird, ist Provenienzforschung aktueller denn je.

\section{Schluss}

Die Konferenzteilnahme in rein digitaler Umgebung blieb auch nach über einem Jahr Pandemieerfahrung gewöhnungsbedürftig. So ließ die Software offenbar nur einen Login zugleich zu, so dass die Verbindung sofort beendet wurde, wenn man an einem „Second Screen“, einem zweiten Gerät, einen kurzen Blick ins Programm werfen wollte. Von solchen Unannehmlichkeiten abgesehen funktionierte die technische Umsetzung aber einwandfrei. $\mathrm{Zu}$ kurz kam hingegen die Möglichkeit, sich mit Kolleginnen und Kollegen zu vernetzen, was ja eigentlich, neben den inhaltlichen Impulsen, eine Hauptfunktion der ganzen Veranstaltung sein sollte. Speziell für Neulinge war es praktisch unmöglich, sich auszutauschen und zu vernetzen. Die sich in den Chats mitunter entspinnenden Anschluss- und Paralleldiskussionen waren zwar inhaltlich oft interessant, aber kein adäquater Ersatz für das Gespräch in der Kaffeepause. Insofern bleibt dringend zu hoffen, dass die Veranstaltung im nächsten Jahr wieder in physischer Präsenz stattfinden kann.

\section{Autoreninformationen}

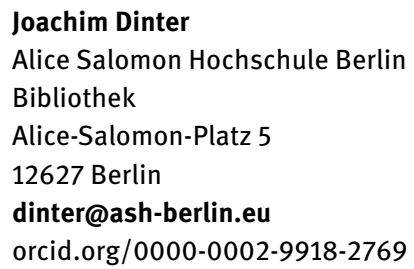

Michael Dominik Hagel

Wissenschaftskolleg zu Berlin

Wallotstraße 19

14193 Berlin

hagel@wiko-berlin.de

orcid.org/0000-0002-8850-5622

\section{Franziska Harnisch}

Universitätsbibliothek der Freien Universität Berlin

Garystraße 39

14195 Berlin

f.harnisch@fu-berlin.de

orcid.org/0000-0002-7863-1836

\section{Tabea Klaus}

Zentral- und Landesbibliothek Berlin

Breite Straße 30-36

10178 Berlin-Mitte

tabea.klaus@zlb.de

orcid.org/0000-0002-2791-1053

\section{Miriam Kötter}

Universitätsbibliothek der Technischen Universität Berlin

Fasanenstraße 88

10623 Berlin

miriambernard@gmx.de

orcid.org/0000-0002-7253-2933

\section{Gunther Kunze}

Ibero-Amerikanisches Institut Preußischer Kulturbesitz Potsdamer Straße 37

10785 Berlin

orcid.org/0000-0002-3715-6178

\section{Mirco Limpinsel-Pesavento}

Universitätsbibliothek der Freien Universität Berlin

Garystraße 39

14195 Berlin

limpinsel@ub.fu-berlin.de

orcid.org/0000-0002-4301-6892

\section{Sina Menzel}

Universitätsbibliothek der Freien Universität Berlin

Garystraße 39

14195 Berlin

sina.menzel@fu-berlin.de

orcid.org/0000-0003-1798-2672

Anne Rethmann

Zentral- und Landesbibliothek Berlin

Breite Straße 30-36

10178 Berlin

anne.rethmann@zlb.de

orcid.org/0000-0001-7632-7550 


\section{Cosima Wagner}

Universitätsbibliothek der Freien Universität Berlin Garystraße 39

14195 Berlin

cosima.wagner@fu-berlin.de orcid.org/0000-0003-4957-3478

\author{
Janet Wagner \\ Universitätsbibliothek der Freien Universität Berlin \\ Garystraße 39 \\ 14195 Berlin \\ janet.wagner@fu-berlin.de \\ orcid.org/0000-0001-6428-1372
}

